\title{
Infant botulism in Singapore
}

Singapore Med J 2020; 61(3): 165-166 https://doi.org/10.11622/smedj.2020017

Dear Sir,

Infant botulism caused by Clostridium botulinum (C. botulinum), an anaerobic, spore-forming Gram-positive bacterium, is rare in Asia. A global study reported 2,932 cases worldwide between 1976 and 2006, with only 25 identified in Asia (22 in Japan, two in China and one in Taiwan). ${ }^{(1)}$ Infant botulism is a diagnostic challenge that frequently results in delay of treatment. Isolation of botulinum toxin (BoNT) in the patient's stool sample via polymerase chain reaction (PCR) can provide a rapid diagnosis. We herein report the first case of infant botulism identified and treated with botulism immune globulin in Singapore.

A nine-month-old boy, the second child of non-consanguineous parents, presented with three days of lethargy, poor suck and decreased feeding. He developed head lag and could not sit unsupported. There was no bowel opening for a week prior to admission. Birth, developmental and immunisation history were normal. Daily examination revealed progressive hypotonia, weakness, bilateral ptosis, weak cry, drooling (oropharyngeal dysphagia) and diminished deep tendon reflexes over the first week of admission. All other systems were unremarkable. Bacterial cultures from urine, blood and cerebrospinal fluid (CSF), viral studies from CSF, urine organic acids, plasma amino acids, serum lactate and ammonia were normal. Magnetic resonance imaging of the brain was normal. Blood and urine toxicology revealed no evidence of drug or heavy metal poisoning.

Supportive care included oropharyngeal suctioning, nasogastric tube feeding and rectal washouts. The child required continuous positive airway pressure, then bilevel positive airway pressure, on Days 3 and 6 of hospitalisation for recurrent desaturations. He was nursed in the intensive care unit with chest physiotherapy and neurorehabilitation. The combination of constipation, acute flaccid paralysis and bulbar weakness raised the differential diagnosis of botulism despite the absence of risk factors. Stool BoNT PCR was positive for C. botulinum toxin A on Day 7, verified by a second sample on Day 11 of hospitalisation.

The patient was treated with $50 \mathrm{mg} / \mathrm{kg}$ human botulism immune globulin (BabyBIG®); procured from the Infant Botulism Treatment and Prevention Program at the California Department of Public Health, California, United States, on Day 16 of hospitalisation. There was gradual improvement in the child's weakness and no further desaturations. Respiratory support was discontinued on Day 25 of hospitalisation, and he was discharged from hospital with nasogastric tube feeding on Day 31.

Botulism is a disease that is legally notifiable to the Ministry of Health, Singapore. A field epidemiology team visited the patient's home to investigate the source of the infection. It led to analysis of the patient's diet, which consisted of breast milk, purees and semi-solid food. Occasionally, commercially available organic baby foods in pre-packaged pouches and probiotic supplements were given but no infant formula or canned/fermented products. Although the patient was not fed honey, his brother consumed honey regularly, and the possibility of accidental ingestion could not be disregarded. The patient also consumed naturopathic sulfur pills prescribed for eczema. The naturopathic pills, Manuka honey, probiotics and pre-packaged food were sent for testing by PCR and bacterial culture, and returned negative for $C$. botulinum and toxins A, B, C, E and F. A definitive source for infection remains unidentified in most cases, as was this case with our patient. ${ }^{(2)}$

Multiplex PCR using the FilmArray® Biothreat Panel (bioMérieux, Marcy-I'Étoile, France) confirmed the presence of BoNT in a stool sample but not in a serum sample. The same result was obtained using a real-time PCR assay. ${ }^{(3)}$ An isolate (designated as BT4) obtained from the stool sample was identified as Clostridium sporogenes by MALDI-TOF (matrix-assisted laser desorption/ ionisation-time of flight) mass spectrometry. This species shares a high similarity with $C$. botulinum strains belonging to toxin group A. Multiplex PCR showed that Type A and B BoNT genes were present in this suspected C. botulinum isolate BT4, and this was consistent with the results obtained from the stool sample. ${ }^{(4)}$ Whole genome sequencing confirmed the identity of BT4 as C. botulinum. C. botulinum of BT4 carried both bont/A1 and bont/B5 genes in a possible bivalent Type Ab configuration. The bont/B5 gene had a single-nucleotide polymorphism that was predicted to result in a premature stop codon. Consistent with the genome data, enzyme-linked immunosorbent assay (ELISA) detected only BoNT/A neurotoxin confirming the expression of the bont/A gene but not BoNT/B. Nevertheless, as expression of neurotoxins up to 24:1 ratio has been reported, the ELISA results do not completely rule out the expression of BoNT/B neurotoxin if the expression of BoNT/B in the samples was too low to be detected by ELISA. ${ }^{(5)}$

Ingested C. botulinum spores germinate in infants' intestines and release the neurotoxins into the bloodstream, where they migrate to the neuromuscular junction. ${ }^{(6)}$ Release of acetylcholine into the synaptic cleft is inhibited, producing flaccid paralysis. Recovery requires regeneration of the neural end plate, which takes several weeks. Botulism immune globulin neutralises the neurotoxin and prevents further deterioration but does not reverse affected neurons. Early administration (preferably within 72 hours of onset of symptoms) of the immune globulin has been shown to reduce duration of hospital stay, mechanical ventilation and tube/intravenous feeding in a randomised trial. ${ }^{(7)}$ The biphasic deterioration in respiratory status in our patient was attributed to ongoing release of toxins, prompting clinicians to treat with botulism immune globulin despite a lack of evidence of efficacy beyond the first week of illness. Even though our patient received botulism immune globulin after two weeks of illness, he showed a satisfactory response to treatment with improvement in weakness and respiratory status. 
The absence of previous cases in Singapore could be due to incomplete surveillance or underdiagnosis. Indeed, a second case of infant botulism was diagnosed in our hospital two months later. Due to heightened awareness of botulism, this case was rapidly diagnosed and botulism immune globulin was administered on Days 6 and 9 of hospital admission, respectively, resulting in a shorter hospital stay (20 days). In conclusion, our case demonstrates that a low threshold for suspicion of botulism is essential despite its rarity in Singapore and that botulism immune globulin is possibly efficacious beyond the first week of illness. Further studies are needed to confirm this.

Yours sincerely,

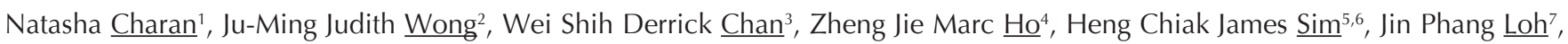
Ling Yann $\underline{\mathrm{FoO}}^{7,8}$, Sophie $\underline{\text { Octavia }}^{9}$, Michelle $\underline{\text { Ang }}^{9}$, Koh Cheng $\underline{\text { Thoon }}^{10}$

${ }^{1}$ Department of Paediatrics, ${ }^{2}$ Children's Intensive Care Unit, ${ }^{3}$ Paediatric Neurology, KK Women's and Children's Hospital, ${ }^{4}$ Communicable Diseases Division, Ministry of Health, ${ }^{5}$ Department of Microbiology, Singapore General Hospital, ${ }^{6}$ Duke-NUS Medical School, ${ }^{7}$ Biological Defence Programme, ${ }^{8} \mathrm{Chemical}$, Toxins, Radiological and Nuclear Defence Programme, DSO National Laboratories, ${ }^{9}$ ational Public Health Laboratory, Ministry of Health, ${ }^{10} \mathrm{Paediatric}$ Infectious Disease, KK Women's and Children's Hospital, Singapore. natasha.charan@mohh.com.sg

\section{Acknowledgement}

We thank Dr Mok Yee Hui and Dr Chong Chia Yin, KK Women's and Children's Hospital, Singapore, for clinical contributions; as well as Dr Matthias Maiwald, KK Women's and Children's Hospital, Singapore; Dr Raymond Lin, National Public Health Laboratory, Ministry of Health, Singapore; Dr Vernon Lee, Communicable Diseases Division, Ministry of Health, Singapore; and Dr Cui Lin, National Public Health Laboratory, Ministry of Health, Singapore, for their epidemiology and laboratory contributions.

\section{References}

1. Koepke R, Sobel J, Arnon SS. Global occurrence of infant botulism, 1976-2006. Pediatrics 2008; 122:e73-82.

2. Rosow LK, Strober JB. Infant botulism: review and clinical update. Pediatr Neurol 2015; 52:487-92.

3. Fohler S, Discher S, Jordan E, et al. Detection of Clostridium botulinum neurotoxin genes (A-F) in dairy farms from Northern Germany using PCR: a case-control study. Anaerobe 2016; 39:97-104.

4. De Medici D, Anniballi F, Wyatt GM, et al. Multiplex PCR for detection of botulinum neurotoxin-producing clostridia in clinical, food, and environmental samples. Appl Environ Microbiol 2009; 75:6457-61.

5. Barash JR, Arnon SS. A novel strain of Clostridium botulinum that produces type B and type H botulinum toxins. J Infect Dis 2014; $209: 183-91$.

6. Sobel J. Botulism. Clin Infect Dis 2005; 41:1167-73.

7. Arnon SS, Schechter R, Maslanka SE, Jewell NP, Hatheway CL. Human botulism immune globulin for the treatment of infant botulism. N Engl J Med 2006; $354: 462-71$. 